\title{
Preparation of flocculant for optimizing glycol lignin manufacturing process by cationization of glycol lignin
}

\author{
Yasuyuki Matsushita ${ }^{1}$ (D) Hiroki Endo ${ }^{1} \cdot$ Shiho Takahashi ${ }^{2} \cdot$ Dan Aoki $^{1} \cdot$ Kazuhiko Fukushima $^{1} \cdot$ Tatsuhiko Yamada $^{2}$
}

Received: 28 February 2018 / Accepted: 11 July 2018 / Published online: 1 August 2018

(c) The Japan Wood Research Society 2018

\begin{abstract}
Glycol lignin possessing thermoplastic and fusible properties can be a biorefinery platform material; however, glycol lignin is obtained as very small particles in suspension and the low dewatering efficiency is a major difficulty for the mass production. To improve the productivity of glycol lignin in the refinery plant, glycol lignin-based flocculants were prepared and their flocculation performance was examined. The flocculants (cationized glycol lignins) were synthesized by a reaction with glycidyltrimethylammonium chloride (GTA) in various reaction conditions. The prepared flocculants exhibited high flocculation performance and successfully economized the production cost of glycol lignin. Their flocculation efficiency was affected by the precipitation temperature, the length of the glycol chain, the molecular weight and the functional group of the glycol lignin. The flocculation behavior was interpreted by phase separation theory at a lower critical solution temperature (LCST).
\end{abstract}

Keywords Lignin · Polyethylene glycol (PEG) · Acid-catalyzed solvolysis · Flocculant · Glycidyltrimethylammonium chloride (GTA)

\section{Introduction}

The Paris agreement was adopted in COP21, and limiting the availability of petroleum chemicals was commonly accepted to prevent global warming. Lignocellulose is an alternative to fossil resources that is expected to provide a solution to the global warming problem, given its carbon neutrality. Lignocellulose materials are mainly composed of two parts: polysaccharides and lignin. Currently, large amounts of polysaccharides are used for the industrial preparation of materials like paper. On the other hand, effective utilization of lignin is yet to be achieved. Thus, lignin is a key material to promote a sustainable social system. Large amounts of lignin (an estimated $2 \times 10^{10}$ t per year) are biosynthesized

Electronic supplementary material The online version of this article (https://doi.org/10.1007/s10086-018-1749-1) contains supplementary material, which is available to authorized users.

Yasuyuki Matsushita

ysmatsu@agr.nagoya-u.ac.jp

1 Graduate School of Bioagricultual Sciences, Nagoya University, Furo-cho Chikusa-ku, Nagoya, Aichi 464-8601, Japan

2 Forestry and Forest Products Research Institute, 1 Matsunosato, Tsukuba, Ibaraki 305-8687, Japan annually, and the excess that is currently leached is $\sim 3 \times 10^{11}$ metric tons [1].

To utilize lignin, separation treatments, such as pulping procedures, are required for lignocellulosic materials. Lignin is connected to polysaccharides through the lignin-carbohydrate complex linkage. The chemical structure of the obtained lignin (technical lignin) is affected in various degrees by the chemical reactions during the separation from lignocellulose; thus, the chemical structure and properties of technical lignins are dependent on reaction conditions. For example, lignin is depolymerized to fragments during Kraft pulping with large chemical changes. Lignosulfonate obtained by sulfite pulping includes sulfonate groups, which contribute to its properties as a polyelectrolyte [2]. Kubo et al. reported the solvolysis of wood meal using carbonate/ethylene glycol with $\mathrm{H}_{2} \mathrm{SO}_{4}$ at $150{ }^{\circ} \mathrm{C}$. The polysaccharides are converted into water-soluble low-molecular-weight compounds, while high-molecular-weight glycol lignin was obtained by grafting glycols into lignin as water-insoluble solids [3]. Ethylene glycol chains were introduced on lignin at $\alpha$ and $\gamma$-positions.

With respect to molding and processing, fusibility is an essential property. However, several technical lignins lack thermal flow properties. The addition of plasticizers, such as poly(ethylene oxide), poly(ethylene terephthalate), and 
polypropylene, allows technical lignin to possess thermal fluidity [4-6]. An acidic solvolysis system using polyethylene glycol (PEG) was also developed [7-9]. In this system, PEG is introduced into lignin to yield polyethylene-modified lignin (glycol lignin), in which the glycol lignin exhibited a viscous thermal flow property [10]. Lin obtained thermally fusible lignin from softwood by cooking a mixture of PEG and sulfuric acid. The fusibility was caused by the PEG moiety introduced by solvolysis. Carbon fibers were prepared from this by melt-spinning followed by oxidative thermostabilization and carbonization procedures [8]. Flexible clay polymer films were prepared by combining glycol lignin and natural clays. The films exhibit a high moisture-barrier property and can be used for flexible electronic devices, such as tough sensors [11, 12].

The glycol lignin is separate from pulp by dissolving it in an alkali solution after acidic solvolysis. The acidity of the solution is adjusted with sulfuric acid to produce a precipitate, and this is centrifuged to obtain solid glycol lignin. In the precipitation process, the glycol lignin is generated as very small particles leading to dewatering difficulties in the centrifuging process. This leads to a reduction in productivity.

In this study, the addition of flocculant to improve dewatering efficiency was investigated. To simplify the system, the flocculant was synthesized from glycol lignin (Fig. 1). Additionally, the use of sulfuric acid and the amount of sodium sulfate as a by-product are reduced if solid glycol lignin is obtained at neutral $\mathrm{pH}$ at the precipitation stage using the flocculant, and this reduces the production cost. In the study, the flocculants were developed for achieving high flocculation efficiency at neutral $\mathrm{pH}$.

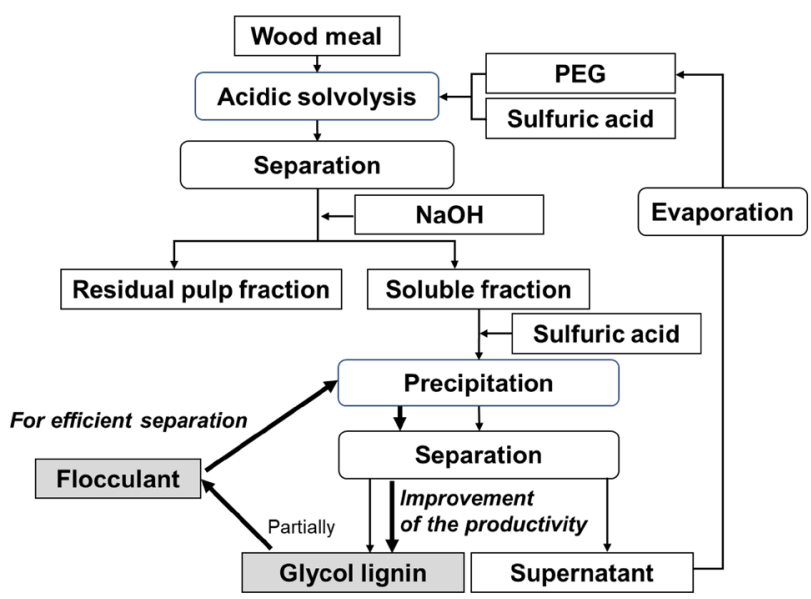

Fig. 1 Schematic illustration for preparation of flocculant with high performance from glycol lignin to improve the production efficiency of glycol lignin [9]

\section{Materials and methods}

\section{Materials}

The glycol lignin was supplied from the bench scale plant of Forestry and Forest Products Research Institute of Japan (FFPRI) which performed an acid catalyzed PEG solvolysis of Japanese cedar (Cryptomeria japonica). The basic process of the glycol lignin production was followed by the previous report [10] with the reaction conditions of $140^{\circ} \mathrm{C}$ of the reaction temperature and $90 \mathrm{~min}$ of the reaction time with acidified PEG $(0.3 \%$ (w/w) sulfuric acid of PEG). In this study, PEG200, PEG400 and PEG600 were used to prepare the glycol lignins (PEG200-lignin, PEG400-lignin and PEG600-lignin, respectively).

\section{Fractionation of glycol lignin}

Specifically, PEG200-lignin was fractionated by gradient acid precipitation [13]. Additionally, PEG200-lignin (20 g) was dissolved in $0.5 \mathrm{~N} \mathrm{NaOH}(1.25 \mathrm{~L})$. A part of glycol lignin was precipitated by adding $0.5 \mathrm{M} \mathrm{H}_{2} \mathrm{SO}_{4}$ dropwise to $\mathrm{pH} 10$. The solution was centrifuged at $10,000 \mathrm{rpm}$ for 10 min to obtain the precipitant (Fraction 1). The supernatant was submitted for a successive precipitation process at a gradient lower $\mathrm{pH}(\mathrm{pH} 9.5,7$, and 2$)$ by the same procedure as mentioned above. The precipitants were termed as Fraction 2, 3 and 4, respectively.

\section{Molecular weight distribution}

The molecular weight of glycol lignin was evaluated using gel permeation chromatography (GPC). The solid phase and elution solution were Sepharose CL-6B and 0.5 N $\mathrm{NaOH}$, respectively. Polystyrene sulfonates were used as standard samples $[14,15]$.

\section{FT-IR spectrometry}

The FT-IR spectra were recorded on a Horiba FT-720 spectrophotometer by the $\mathrm{KBr}$ method.

\section{Preparation of the flocculant from glycol lignin by cationization}

Furthermore, glycol lignin was dissolved in $0.5 \mathrm{~N} \mathrm{NaOH}$, and glycidyltrimethylammonium chloride (GTA) was added to the solution. It was heated at $50{ }^{\circ} \mathrm{C}$ for $3 \mathrm{~h}$. The reaction mixture was dialyzed using a cellulose tube (3500 MWCO) or neutralization with $\mathrm{H}_{2} \mathrm{SO}_{4}$ [16]. Nitrogen 
Table 1 Reaction conditions for preparation of cationized glycol lignin and nitrogen content of the cationized glycol lignin

\begin{tabular}{|c|c|c|c|c|c|}
\hline \multirow{3}{*}{$\begin{array}{l}\text { Cationized } \\
\text { glycol lignin }\end{array}$} & \multicolumn{4}{|c|}{ Reaction conditions } & \multirow{3}{*}{$\begin{array}{l}\begin{array}{l}\text { Reaction } \\
\text { product }\end{array} \\
\mathrm{N} \text { content } \\
(\%)\end{array}$} \\
\hline & \multicolumn{2}{|l|}{ Raw material } & \multirow{2}{*}{$\begin{array}{l}\text { GTA } \\
(\mathrm{mL})\end{array}$} & \multirow{2}{*}{$\begin{array}{l}\text { Treatment } \\
\text { after reaction }\end{array}$} & \\
\hline & Glycol lignin & (g) & & & \\
\hline P200G1 & $\begin{array}{l}\text { PEG200- } \\
\text { lignin }\end{array}$ & 1.0 & 3.0 & Dialysis & 2.3 \\
\hline P200G2 & $\begin{array}{l}\text { PEG200- } \\
\text { lignin }\end{array}$ & 1.0 & 1.0 & Dialysis & 1.7 \\
\hline P200G3 & $\begin{array}{l}\text { PEG200- } \\
\text { lignin }\end{array}$ & 3.0 & 1.0 & Dialysis & 1.5 \\
\hline P200G4 & $\begin{array}{l}\text { PEG200- } \\
\text { lignin }\end{array}$ & 3.0 & 0.5 & Dialysis & 1.1 \\
\hline P200G5 & $\begin{array}{l}\text { PEG200- } \\
\text { lignin }\end{array}$ & 3.0 & 0.2 & Dialysis & 0.4 \\
\hline P200G6 & $\begin{array}{l}\text { PEG200- } \\
\text { lignin }\end{array}$ & 3.0 & 1.0 & $\begin{array}{l}\text { Neutraliza- } \\
\text { tion }\end{array}$ & 1.8 \\
\hline P400G & $\begin{array}{l}\text { PEG400- } \\
\text { lignin }\end{array}$ & 3.0 & 1.0 & $\begin{array}{l}\text { Neutraliza- } \\
\text { tion }\end{array}$ & 2.0 \\
\hline P600G & $\begin{array}{l}\text { PEG600- } \\
\text { lignin }\end{array}$ & 3.0 & 1.0 & $\begin{array}{l}\text { Neutraliza- } \\
\text { tion }\end{array}$ & 2.0 \\
\hline $\mathrm{F} 1 \mathrm{G}^{\mathrm{a}}$ & Fraction $1^{\mathrm{a}}$ & 0.2 & 0.2 & $\begin{array}{l}\text { Neutraliza- } \\
\text { tion }\end{array}$ & 3.5 \\
\hline $\mathrm{F} 2 \mathrm{G}^{\mathrm{a}}$ & Fraction $2^{\mathrm{a}}$ & 0.2 & 0.2 & $\begin{array}{l}\text { Neutraliza- } \\
\text { tion }\end{array}$ & 3.5 \\
\hline $\mathrm{F} 3 \mathrm{G}^{\mathrm{a}}$ & Fraction $3^{\mathrm{a}}$ & 0.2 & 0.2 & $\begin{array}{l}\text { Neutraliza- } \\
\text { tion }\end{array}$ & 3.5 \\
\hline $\mathrm{F} 4 \mathrm{G}^{\mathrm{a}}$ & Fraction $4^{\mathrm{a}}$ & 0.2 & 0.2 & $\begin{array}{l}\text { Neutraliza- } \\
\text { tion }\end{array}$ & 3.9 \\
\hline
\end{tabular}

${ }^{\text {a }}$ PEG200-lignin was fractionated by gradient acid precipitation at pH 10 (Fraction 1), pH 9.5 (Fraction 2), pH 7 (Fraction 3) and pH 2 (Fraction 4). F1G, F2G, F3G and F4G were prepared from Fraction 1, 2,3 and 4 , respectively

content was determined by performing an elementary analysis. The reaction conditions are shown in Table 1.

\section{Flocculation test}

PEG400-lignin was used for the flocculation test as a suspension ( $\mathrm{pH}$ 6.5) including small particles of glycol lignin. The required amount of the cationized glycol lignin $(0.1,0.5$, and $1.0 \mathrm{wt} \%$ based on solid content of the suspension) was added to the suspension in a test tube, and the cap was closed. After turning it upside down immediately once, the volume of the precipitate was measured with respect to time as follows:

Volume of the precipitate $(\%)=\frac{b}{a} \times 100$,

where $b$ denotes the height of flocculated glycol lignin in the test tube, and $a$ denotes the height of the suspension solution in the test tube prior to adding the flocculant. Essentially, the tests were operated at $50{ }^{\circ} \mathrm{C}$ and $\mathrm{pH}$ 6.5. To
Table 2 Molecular weight and proportion of glycol lignins

\begin{tabular}{lll}
\hline & Proportion $(\%)$ & $M_{\mathrm{w}}\left(\times 10^{3} \mathrm{~g} / \mathrm{mol}\right)$ \\
\hline PEG200-lignin & - & 6.5 \\
PEG400-lignin & - & 8.1 \\
PEG600-lignin & - & 8.0 \\
Fraction 1 & 86.9 & 9.7 \\
Fraction 2 & 3.9 & 8.0 \\
Fraction 3 & 5.4 & 6.2 \\
Fraction 4 & 3.7 & 4.9 \\
\hline
\end{tabular}

investigate the effect on the temperature, the experiment was performed at 30 and $50{ }^{\circ} \mathrm{C}$.

\section{Results and discussion \\ Properties of glycol lignins}

In this study, glycol lignins (PEG200-lignin, PEG400-lignin, and PEG-600-lignin) were used. Additionally, four fractionated PEG200-lignins that were separated by gradient acid precipitation were also submitted to prepare the flocculant. Table 2 shows the molecular weight and proportion of the fractions, and the GPC profiles are shown in Fig. S1 and Fig. S2. The molecular weight of PEG200-lignin was lower than that of PEG400-lignin and PEG600-lignin. There is a low difference in molecular weight between PEG400-lignin and PEG600-lignin. Given the characteristics of gel permeation chromatography, both the hydrodynamic radii may exhibit almost the same value.

There was a high difference in molecular weight between the four fractions, and this suggested that high molecular weight glycol lignin was precipitated at high $\mathrm{pH}$. A potential reason is that the range of van der Waals attractive forces of large molecules exceeds that of small molecules [13]. The molecular weight of Fraction 1 was approximately twice higher than that of fraction 4 . The proportion of Fraction 1 was $86.9 \%$, and this suggested that it was a major part of PEG200-lignin.

Figure 2 shows the IR spectra of the glycol lignins. In all the glycol lignins, the peak at $2800-3000 \mathrm{~cm}^{-1}$ attributed to the stretching of the methylene group was observed. The highest intensity corresponded to the peak for PEG600lignin due to the presence of a high amount of ethylene oxide unit. In the spectrum of PEG200-lignin, the band at $1716 \mathrm{~cm}^{-1}$ appears due to the stretching of the carbonyl group of carboxylic acid. The peak was not observed in the spectra of Fraction 1, 2 and 3. However, only Fraction 4 that was precipitated at $\mathrm{pH} 2$ exhibited a carboxylic acid peak. This implied that the carboxylic acid group was contained in the small molecules of glycol lignin. 


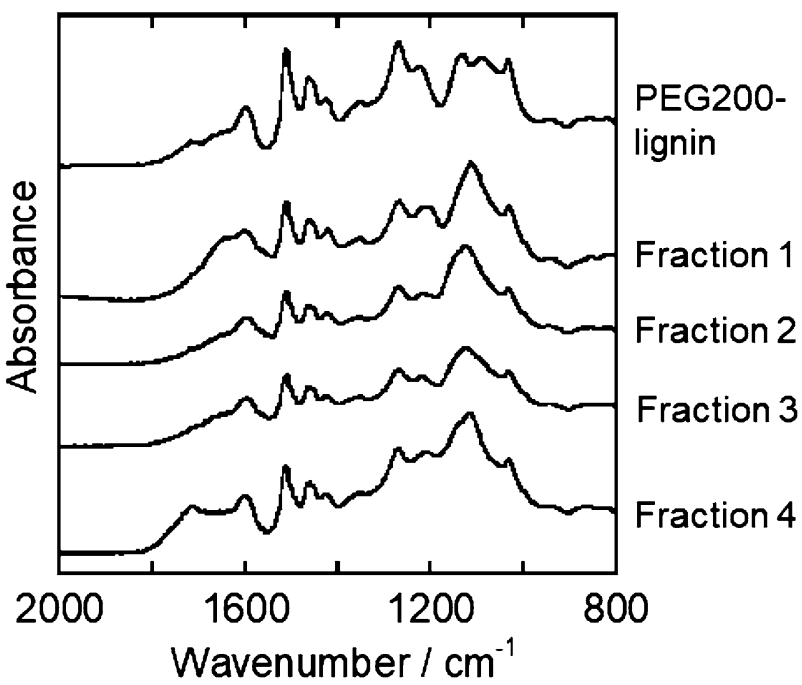

Fig. 2 FT-IR spectra of PEG-lignin and Fraction 1-4

\section{Preparation of flocculant from glycol lignin}

In the system, it is observed that the cationic polymer is adequate for the flocculant because glycol lignin includes a phenolic hydroxyl group. Specifically, lignosulfonate includes anionic groups although it is not efficient as a flocculant. In our study, a dimethylamino group was introduced into phenolated sulfuric acid lignin by the Mannich reaction [17-19].

GTA was also used as a cationization reagent. Generally, cationic starch that is produced by a reaction between starch and GTA is routinely added to a wet-end process to improve the dry strength of paper [20]. Seong et al. synthesized chito-oligosaccharide using GTA as antimicrobial agent [21]. Wahlström et al. prepared cationic lignins from hardwood organosolv lignin and enzymatic hydrolysis lignin through a reaction with GTA and applied the same to water purification [22].

The quaternary ammonium derivatives steadily exhibit a cationic property in the aqueous solution independent of the $\mathrm{pH}$. Thus, in this study, GTA was selected to introduce the cationic group on glycol lignin. The reaction mechanism is shown in Fig. 3. The phenolic hydroxyl group is reacted with GTA [23]. Additionally, PEG exhibits high values of the second virial coefficient and low values of the polymer-solvent interaction parameter $(\chi)$, and this suggests that PEG chain of glycol lignin exhibits high mobility and high excluded volume [24]. Thus, there is a possibility that the hydroxy group at the end of the long-chain PEG moiety can react with GTA because the moiety spreads widely in the solution and results in GTA readily participating in the reaction.

As a competitive reaction, GTA was reacted with hydroxy ions. The proportion of this side reaction increases when the concentration of hydroxyl ion is high. In the study, $0.5 \mathrm{~N}$ $\mathrm{NaOH}$ was selected based on a previous study [22].

Table 1 shows the reaction conditions and nitrogen contents of the reaction products. The nitrogen content in cationized PEG200-lignin was 2.3\% when excess GTA was reacted (P200G-1 in Table 1). As expected, the nitrogen content decreased with decreases in the dosage proportion of GTA to PEG200-lignin (P200G1-P200G5 in Table 1). When an excessively low amount of GTA is added, it causes insolubility of the product in water at neutral pH (P200G4 and
Fig. 3 Proposed reaction mechanism for a cationization of glycol lignin and $\mathbf{b}$ side reaction using GTA (a)

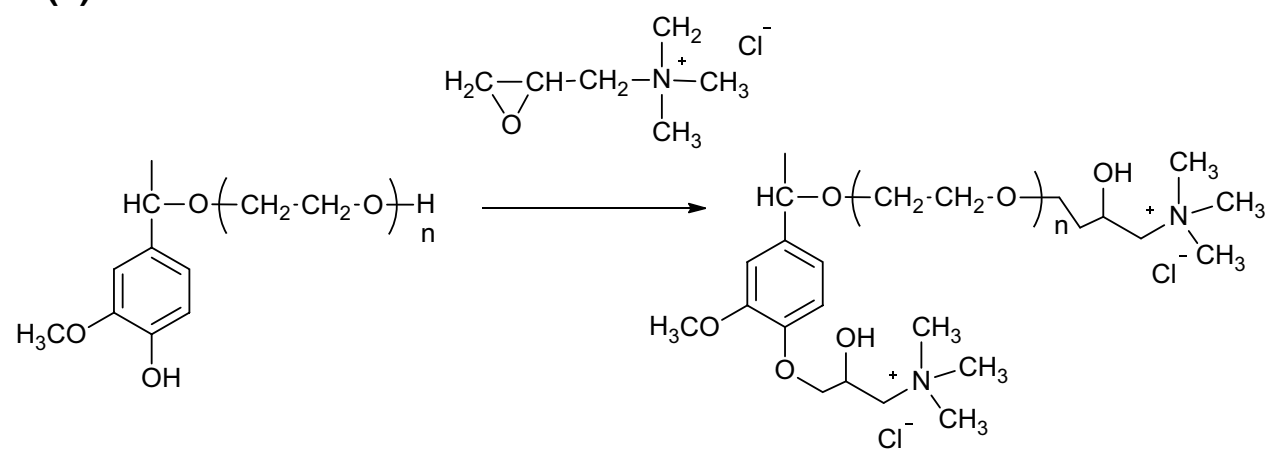<smiles>C[N+](C)(C)CC(O)COC(O)CC1CO1</smiles> 
P200G5 in Table 1). The results indicate that the minimum amount of GTA required to dissolve the reaction product into water is $1 \mathrm{ml}$ for $3 \mathrm{~g}$ of PEG200-lignin (P200G3, nitrogen content is $1.5 \%$ ).

To shorten work hours and reduce the number of the operation processes for preparing the flocculant, neutralization was applied as opposed to dialysis. With respect to the nitrogen content of the reaction product in P200G3 and P200G6, both exhibited almost similar values. This suggested that the amount of the remaining side reaction products was low in the reaction conditions of P200G3 and P200G6, and thus they did not significantly affect the performance of the prepared flocculant.

\section{Flocculation test}

\section{Effect of reaction conditions}

In the suspension solution, the surface charge of glycol lignin exhibited a negative value due to the phenolic hydroxy group. Thus, the prepared cationized glycol lignin acts as a flocculant for the small particle glycol lignin through an electrostatic interaction. In the study, the flocculation efficiency was estimated by measuring the volume of the precipitate after the addition of the cationized glycol lignin.

To investigate the effect on the flocculation efficiency of temperature, P200G1 submitted the flocculation test at 30 and $50{ }^{\circ} \mathrm{C}$ (Fig. 4). The results suggested that the flocculation efficiency increased with increases in the temperature. This phenomenon could be related to the lower critical solution temperature (LCST) of glycol lignin. Generally, with respect to the LCST, the micelle of nonionic surfactants with a polyethylene glycol chain exhibits phase separation due to the cleavage of the hydrogen bond between polyethylene

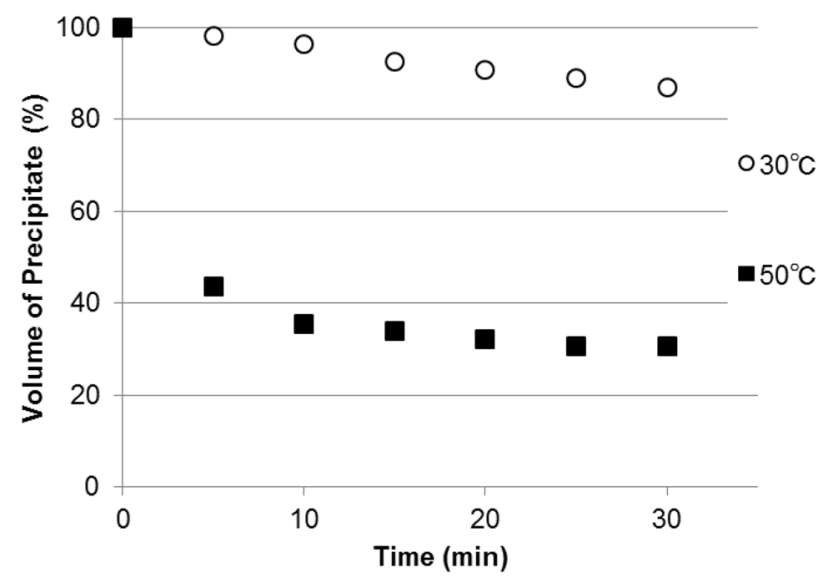

Fig. 4 Effect on flocculation efficiency of P200G1 at different temperature glycol chain and water, and this lead to a decrease in the solubility [25].

In the study, when the prepared flocculant was added to the suspension of glycol lignin, the trimethylamino group of the prepared flocculant connected to the glycol lignin to generate a complex. The complex (glycol lignin-cationized glycol lignin) included long chain PEG regions at the surface that act as the nonionic surfactant. An increase in the temperature allowed the complex to easily agglomerate with each other due to the breakage of the hydrogen bonds between the complex and water.

Figure 5 shows the volume of the precipitate in P200G1-P200G3 and P200G6 at $50{ }^{\circ} \mathrm{C}$ and pH 6.5. All prepared flocculants exhibited the flocculation ability when the dosage was $1 \mathrm{wt} \%$. At $0.5 \mathrm{wt} \%$ dosage, P200G1 exhibited flocculation performance given its high nitrogen content although the other flocculants did not exhibit the same. When the dosage was $0.1 \mathrm{wt} \%$, all prepared flocculants did not exhibit flocculation performance for the flocculation.

The flocculant efficiency of P200G6 has higher than that of P200G2, even if the nitrogen contents of both are same (Fig. 5b, d; Table 1). The difference might be caused by the preparation procedure; however, it is difficult to show clearly why P200G2 was better.

According to Fig. 5c, d, neutralization was better than dialysis for the posttreatment of the preparation reaction. The cationized glycol lignin had high flocculation performance even if the posttreatment was neutralization. It leads the simplicity of the production process for the cationized glycol lignin, resulting in the increase of productivity for glycol lignin.

\section{Effect of the length of PEG in glycol lignin}

The hydrophilic PEG chains in glycol lignin were considered as spread widely in the solution, and this affected the flocculation efficiency of the cationized glycol lignin. Figure 6 shows the flocculation efficiency of the prepared cationized glycol lignin using PEG200-lignin, PEG400lignin, and PEG600-lignin. The efficiencies of the cationized PEG200-lignin (P200G6) and PEG400-lignin (P400G) were equivalent to each other while the cationized PEG600lignin (P600G) exhibited lower efficiency when compared with those of the others. This is also explained by the LCST theory. The amount of hydrogen bonds between the PEG chain of the coagulation complex (glycol lignin-cationized glycol lignin) and water increased with increases in the length of PEG chain, and thereby led to increases in the LCST. Thus, it was difficult for the complexes including cationic PEG600-lignin to agglomerate with each other. This phenomenon was also observed in the water-soluble polymer with PEG (co-polymer using $N$-isopropyl acryl amide and polyethylene glycol with primary amine). Hazer 

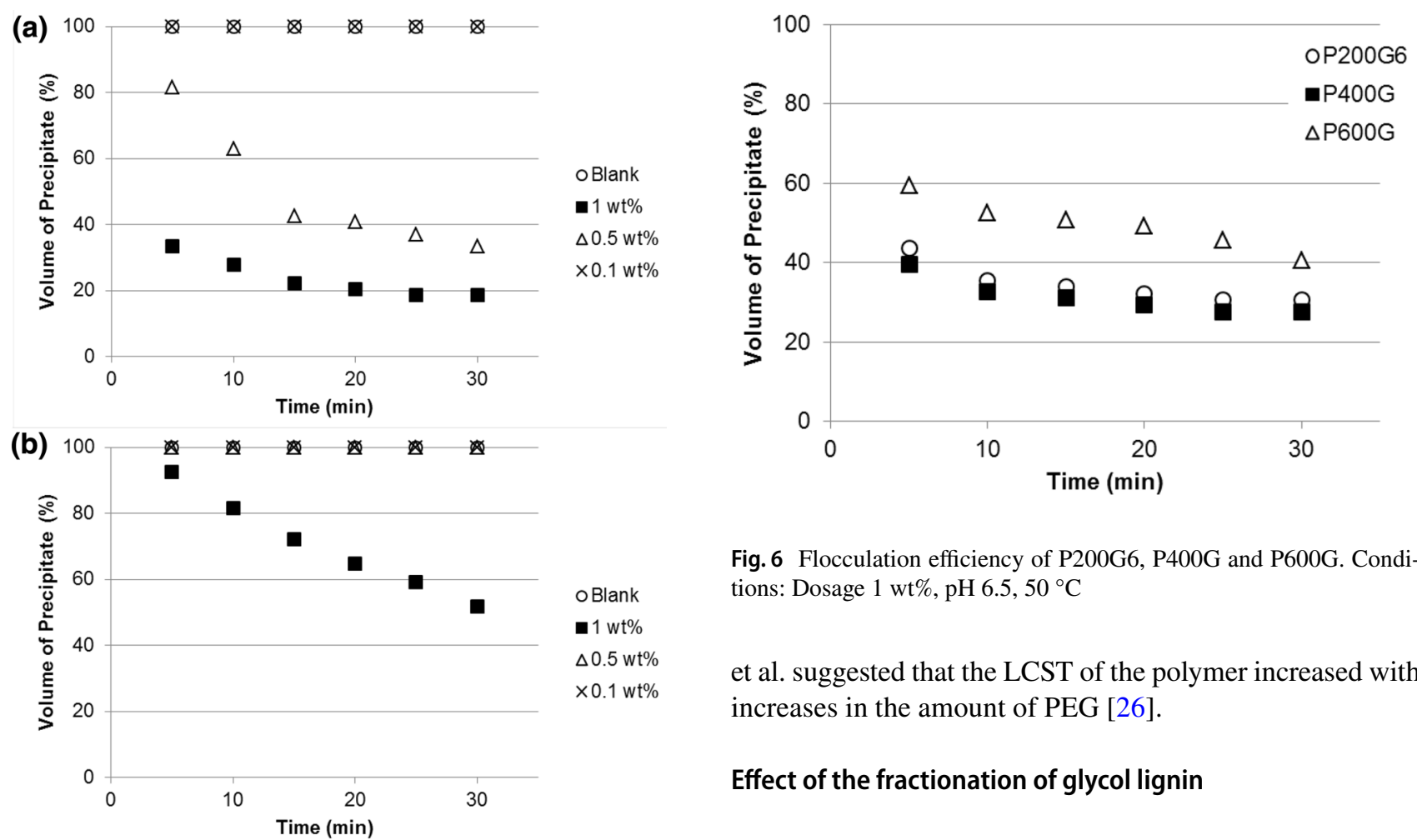

Fig. 6 Flocculation efficiency of P200G6, P400G and P600G. Conditions: Dosage $1 \mathrm{wt} \%, \mathrm{pH} 6.5,50{ }^{\circ} \mathrm{C}$

et al. suggested that the LCST of the polymer increased with increases in the amount of PEG [26].

\section{Effect of the fractionation of glycol lignin}

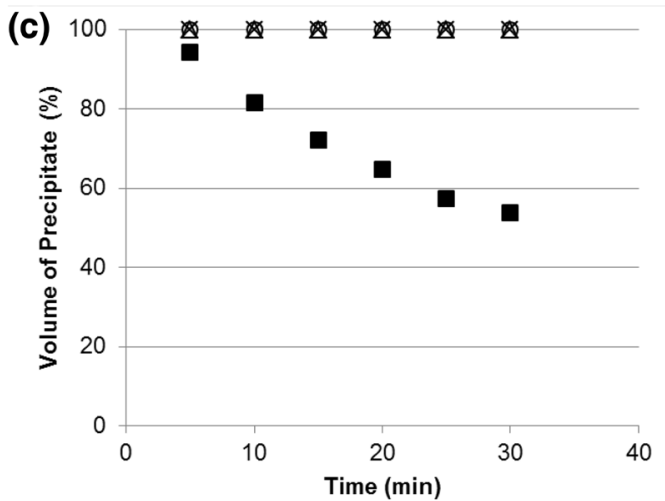

o Blank

- $1 \mathrm{wt} \%$ $\Delta 0.5 \mathrm{wt} \%$ $\times 0.1 w t \%$

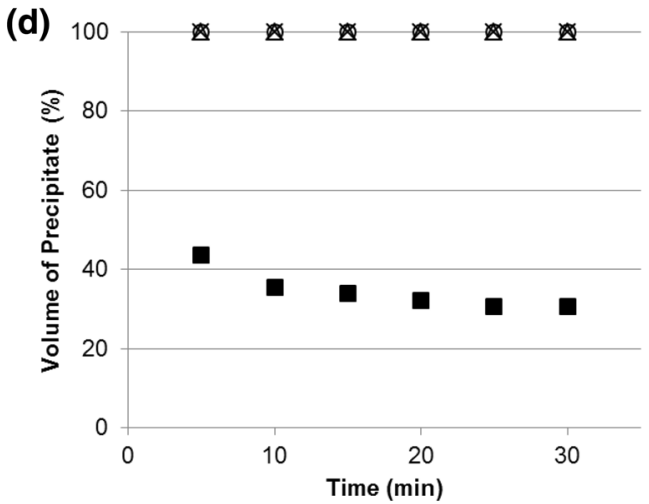

Fig. 5 Volume of precipitate flocculation test. a P200G1 b P200G2 c P200G3 d P200G6. Conditions: Dosage 0.1, 0.5 and $1 \mathrm{wt} \%, 50{ }^{\circ} \mathrm{C}$, and $\mathrm{pH} 6.5$$$
\text { - } 1 \mathrm{wt} \%
$$
$\times 0.1 \mathrm{wt} \%$

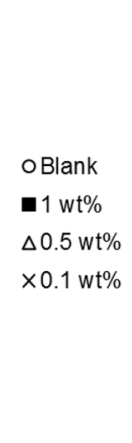

\section{OBlank}

To investigate the characteristics of the glycol lignin for cationization, fractionated PEG200-lignins (F1G, F2G, $\mathrm{F} 3 \mathrm{G}$, and F4G in Table 1) were subjected to a reaction with GTA, and the flocculation efficiency was estimated (Fig. 7). F1G-F3G shows the flocculation ability although F4G did not exhibit flocculation. Specifically, F4G included carboxylic acid (Fig. 2) that influenced the surface electrostatic charge of the $\mathrm{F} 4 \mathrm{G}$.

The flocculation efficiency of $F 2 G$ was lower than that of F1G and F3G. The difference might be influenced by many factors, such as the ratio of nitrogen content to molecular

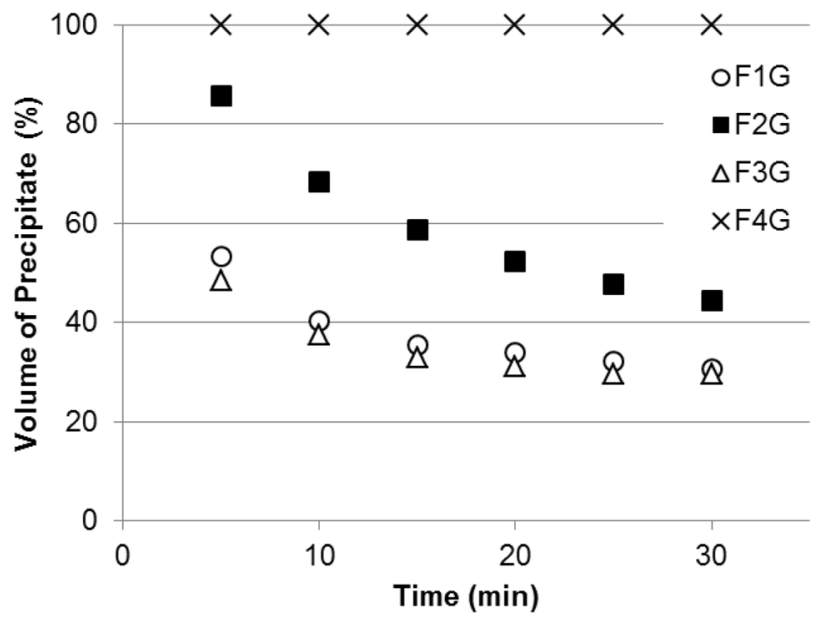

Fig. 7 Flocculation efficiency of F1G-F4G. Conditions: Dosage $1 \mathrm{wt} \%, \mathrm{pH} 6.5$ and $50{ }^{\circ} \mathrm{C}$ 
weight and mass of lignin to PEG moiety. However, it is difficult to mention the reason. It requires further research to understand the flocculation efficiency clearly.

\section{Conclusions}

In this study, lignin-based flocculants were synthesized from glycol lignin through the simple reaction system. Their performance on the glycol lignin production system was examined in consideration of the structural parameter and reaction conditions. As a result, the optimally prepared flocculant showed high flocculation ability and improved the glycol lignin productivity by acceleration of the dewatering process. The improvement of glycol lignin production using glycol lignin-based flocculants should be a favorable selfcooperation approach to realize commercial mass production of the biorefinery platform material.

Acknowledgements This work was supported by Cabinet Office, Government of Japan, Cross-ministerial Strategic Innovation Promotion Program (SIP), "Technologies for creating next-generation agriculture, forestry and fisheries", SIP-Lignin Project (funding agency: Bio-oriented Technology Research Advancement Institution, NARO).

\section{References}

1. Hu L, Pan H, Zhou Y, Zhang M (2011) Methods to improve lignin's reactivity as a phenol substitute and as replacement for other phenolic compounds: a brief review. Bioresour 6:3515-3525

2. Matsushita Y (2015) Conversion of technical lignins to functional materials with retained polymeric properties. J Wood Sci 61:230-250

3. Kubo S, Yamada T, Hashida K, Ono H (2007) Grafting of ethylene glycol chains in lignin during the solvolysis for biomass conversion using ethylene carbonate/ethylene glycol system. Chem Lett 36:502-503

4. Kadla JF, Kubo S, Venditti RA, Gilbert RD, Compere AL, Griffith W (2002) Lignin-based carbon fibers for composite fiber applications. Carbon 40:2913-2920

5. Kubo S, Kadla JF (2005) Kraft lignin/poly(ethylene oxide) blends: effect of lignin structure on miscibility and hydrogen bonding. $\mathrm{J}$ Appl Polym Sci 98:1437-1444

6. Kubo S, Kadla JF (2005) Lignin-based carbon fibers: effect of synthetic polymer blending on fiber properties. J Polym Environ 13:97-105

7. Mörck R, Reimann R, Kringstad KP (1989) Elastomeric polyurethanes from a kraft lignin-polyethylene glycol-toluene diisocyanate system. In: Glasser WG, Sarkanen S (eds) Lignin: properties and materials ACS symposium series 387. American Chemical Society, Washington, DC, pp 390-401

8. Lin J, Kubo S, Yamada T, Koda K, Uraki Y (2012) Chemical thermostabilization for the preparation of carbon fibers from softwood lignin. BioResour 7:5634-5646

9. Takata E, Nge TT, Takahashi S, Ohashi Y, Yamada T (2016) Acidic solvolysis of softwood in recycled polyethylene glycol system. BioRecour 11:4446-4458
10. Nge TT, Takata E, Takahashi S, Yamada T (2016) Isolation and thermal characterization of softwood-derived lignin with thermal flow properties. ACS Sustain Chem Eng 4:2861-2868

11. Kanako H, Ishii R, Suzuki A, Nakamura T, Ebina T, Nge TT, Yamada T (2016) Flexible clay glycol lignin nanocomposite film with heat durability and high moisture-barrier property. Appl Clay Sci 132-133:425-429

12. Takahashi K, Ishii R, Nakamura T, Suzuki A, Ebina T, Yoshida M, Kubota M, Nge TT, Yamada T (2017) Flexible electronic substrate film fabricated using natural clay and wood components with cross-linking polymer. Adv Mater 29:1606512

13. Wang G, Chen H (2013) Fractionation of alkali-extracted lignin from steam-exploded stalk by gradient acid precipitation. Sep Purif Technol 105:98-105

14. Yasuda S, Ito K, Hamaguch E, Matsushita Y (1997) Ready chemical conversion of acid hydrolysis lignin into water-soluble lignosulfonate I. successive treatment of acid hydrolysis lignin by phenolation, hydroxymethylation and sulfonation. Mokuzai Gakkaishi 43:68-74

15. Matsushita Y, Inomata T, Hasegawa T, Fukushima K (2009) Solubilization and functionalization of sulfuric acid lignin generated during bioethanol production from woody biomass. Bioresour Technol 100:1024-1026

16. Matsushita Y, Sekiguchi T, Saito K, Kato T, Imai T, Fukushima $\mathrm{K}$ (2007) The characteristic fragment ions and visualization of cationic starches on pulp fiber using ToF-SIMS. Surf Interface Anal 39:501-505

17. Matsushita Y, Yasuda S (2003) Reactivity of a condensed-type lignin model compound in the Mannich reaction and preparation of cationic surfactant from sulfuric acid lignin. J Wood Sci 49:166-171

18. Matsushita Y, Iwatsuki A, Yasuda S (2004) Application of cationic polymer prepared from sulfuric acid lignin as a retention aid for usual rosin sizes to neutral papermaking. J Wood Sci 50:540-544

19. Matsushita Y, Imai M, Iwatsuki A, Fukushima K (2008) Relationships between surface tension and industrial performance of water-soluble polymers prepared from acid hydrolysis lignin, a saccharification by-product from woody materials. Bioresour Technol 99:3024-3028

20. Matsushita Y, Suzuki A, Sekiguchi T, Saito K, Imai T, Fukushima K (2008) Mapping of the cationic starch adsorbed on pulp fibers by ToF-SIMS. Appl Surf Sci 255:1022-1024

21. Seong H-S, Whang HS, Ko S-W (2000) Synthesis of a quaternary ammonium derivative of chitooligosaccharide as antimicrobial agent for cellulosic fibers. J Appl Polym Sci 76:2009-2015

22. Wahlström R, Kalliola A, Heikkinen J, Kyllönen H, Tamminen T (2017) Lignin cationization with glycidyltrimethylammonium chloride aiming at water purification applications. Ind Crop Prod 104:188-194

23. Matsushita Y, Yasuda S (2003) Preparation of anionic-exchange resins from pine sulfuric acid lignin, one of the acid hydrolysis lignins. J Wood Sci 49:423-429

24. Lee JH, Lee HB, Andrade JD (1995) Blood compatibility of polyethylene oxide surfaces. Prog Polym Sci 20:1043-1079

25. Grinberg VY, Burova TV, Grinberg NV, Dubovik AS, Papkov VS, Khokhlov AR (2015) Energetics of LCST transition of poly(ethylene oxide) in aqueous solutions. Polym 73:86-90

26. Hazer B, Ayyıldız E, Bahadır F (2017) Synthesis of PNIPAMPEG double hydrophilic polymers using oleic acid macro peroxide initiator. J Am Oil Chem Soc 94:1141-1151 